Original Research

\title{
Dichotomous effects of autophagy on infarct volume in experimental permanent/transient ischemic stroke model: a systematic review and meta-analysis
}

\author{
Aysa Rezabakhsh ${ }^{1,2, *, \dagger}$, Nafiseh Vahed $^{3,{ }^{\dagger}}$, Hossein Hosseinifard ${ }^{3, \dagger}{ }^{\dagger}$, Reza Rahbarghazi ${ }^{4,5}$, Fatemeh Salehnia ${ }^{6}$, \\ Yalda Sadeghpour ${ }^{7}$, Sarvin Sanaie ${ }^{7, *}$ \\ ${ }^{1}$ Cardiovascular Research Center, Tabriz University of Medical Sciences, 5166614766 Tabriz, Iran \\ ${ }^{2}$ Emergency Medicine Research Team, Tabriz University of Medical Sciences, 5166614766 Tabriz, Iran \\ ${ }^{3}$ Research Center for Evidence-Based Medicine, Tabriz University of Medical Sciences, 5166614766 Tabriz, Iran \\ ${ }^{4}$ Stem Cell Research Center, Tabriz University of Medical Sciences, 5166614766 Tabriz, Iran \\ ${ }^{5}$ Department of Applied Cell Sciences, Faculty of Advanced Medical Sciences, Tabriz University of Medical Sciences, 5166614766 Tabriz, Iran \\ ${ }^{6}$ Research Development \& Coordination Center, Tabriz University of Medical Sciences, 5166614766 Tabriz, Iran \\ ${ }^{7}$ Neurosciences Research Center, Tabriz University of Medical Sciences, 5166614766 Tabriz, Iran
}

*Correspondence: aysapharma.rezabakhsh@gmail.com; rezabakhsha@tbzmed.ac.ir (Aysa Rezabakhsh); sarvin_sozooo@yahoo.com (Sarvin Sanaie)

${ }^{\dagger}$ These authors contributed equally.

\section{DOI:10.31083/j.jin2101011}

This is an open access article under the CC BY 4.0 license (https://creativecommons.org/licenses/by/4.0/).

Submitted: 7 April 2021 Revised: 17 May 2021 Accepted: 18 August 2021 Published: 28 January 2022

According to the recent findings, autophagy modulation is being a potential therapeutic target in the management of ischemic stroke in a pre-clinical setting. However, the pros and cons of autophagic response strongly depend on the activation time of autophagy after injury. In this systematic review, we aimed to explore the impacts of pharmacological modulation of autophagy on infarct size in experimental ischemic stroke models. Based on our preliminary search, 3551 publications were identified. Of twenty-nine publications that met the inclusion criteria, twenty studies reported infarct volume reduction by percentage (\%) with no evidence of any publication bias while nine studies reported by $\mathrm{mm}^{3}$, which had publication bias (39.25 units, standardized mean differences (SMD) $=41.92,95 \%$ confidence interval $(\mathrm{Cl}): 30.33$ to 53.51). Based on a meta-analysis, the point estimate (pooled mean difference) for improvement of infarct volume during autophagy modulation according to the $\mathrm{mm}^{3}$ and percentage were 35.64 (mean differences $(M D)=35.64,95 \% \mathrm{Cl}$ : 26.43 to $44.85, z$-value $=7.58, p$-value $<0.001)$ and $14.38(M D=14.38$, $95 \% \mathrm{Cl}=10.50$ to $18.26, \mathrm{z}$-value $=7.26, p<0.001$ ) units, respectively. Despite the undeniable role of autophagy in ischemic stroke, the dichotomous effects of autophagy regarding infarct volume reduction should be taken into account. Based on our findings, the studies included in this meta-analysis mostly reported a negative relation between autophagy induction and stroke volume development due to over-activity of autophagy upon the severe ischemic stroke; therefore, further pre-clinical studies are also recommended to establish adjusted autophagy with considering a time-dependent effect as a promising therapeutic target.

Keywords

Autophagy; Dual effects; Ischemic stroke; Pre-clinical setting

\section{Introduction}

Ischemic stroke (IS), one of the devastating disorders, is the intended second leading cause of mortality and disability followed by vascular occlusion and irreversible damage of the brain tissue $[1,2]$. Despite the rising aged population, the incidence of stroke is expected to grow thereby a demand to accede a novel and more effective therapeutic approach is increasing, particularly for patients suffering from acute cerebral ischemia [3, 4]. It has been proved that long-lasting autophagy besides a variety of other neurologic conditions plays a crucial role in cerebral ischemic injury. However, growing pieces of evidence demonstrated that autophagy has the potential to exert controversial effects (either detrimental or beneficial) in cerebral IS [5]. In better words, regulated and moderate autophagy may provide a neuroprotection effect while an excessive or inappropriate activation of autophagy could trigger deleterious effects to develop cell death [6, 7]. Autophagy, a catabolic-conserved process through the breakdown and subsequent recycling of cellular constituents, is an essential physiological intracellular process for maintaining cellular homeostasis and simultaneously participates in bio-energetic procedures under various stress conditions [8]. This phenomenon is highly regulated by numerous molecules such as microtubule-associated protein 1A light chain 3 (LC3), Beclin-1, and P62 (a scaffold protein) that have a necessary role in the regulation of the autophagy signaling pathway [9]. Of note, the excessive activation of autophagy and related effectors in neural cells have been firmly established in a variety of focal ischemic stroke 
models such as experimental middle cerebral artery occlusion (MCAO). Moreover, recent evidence demonstrated that the over-activity of neuronal autophagy through persistent stress, such as cerebral ischemia, results in cell damage, especially in the border area of lesion sites $[10,11]$. Therefore, autophagy regulation could be considered a potential target for IS treatment [12]. In contrast, it has also been reported that pre-activation of autophagy in the brain tissue could enhance brain ischemic tolerance, facilitate cellular energy production, and prevent neuronal apoptosis during subsequent exposure to the ischemic conditions [13]. For instance, rapamycin, as a well-known autophagy inducer has a palliative effect on pre-clinical IS damage through the activation of mitophagy, suggesting that autophagy has a beneficial effect on ischemia/reperfusion injury. Although there is no debate regarding autophagy participation in cerebral ischemia, the accurate function of autophagy in IS remains controversial. In hence, the main purpose of this systematic review refers to uncover a total pattern of infarct volume evolution after autophagy modulation quantitatively via meta-analysis in the experimental models of stroke.

\section{Methods}

\subsection{Search strategy}

For the primary systematic search strategy, Embase, Medline (via PubMed, Ovid) databases were used. Notably, all considered studies were published in English and the inception date of each database was qualified for inclusion in this review (from 1980-Jan till 2021-May). In addition, the search strategy aimed to explore both published and unpublished studies with the combination of Mesh and free keywords such as autophagy, macroautophagy, cerebrovascular accident, ischemic stroke, and autophagy biomarkers. A complete search strategy in the PubMed database is brought in the supplementary material (Appendix Table 3).

\subsection{Inclusion and exclusion criteria}

This quantitative study was deliberated to include all studies calculated infarct size following the assessment of autophagy detrimental and/or protective effects as the primary outcome in the IS model of rodents who underwent experimental transient/prominent ischemia induced by MCAO as well as focal cerebral ischemia. There was not any exclusion based on the route of drug administration, divergent medications used for anesthesia, and the duration of treatment. The full text of selected studies that did not meet the inclusion criteria such as clinical trials, in vitro experiments, nonEnglish written articles, the published conference abstracts, and the articles without standard quality, such as not mentioned quantitative changes in case of the infarct size with percentage or $\mathrm{mm}^{3}$, were ultimately excluded.

\subsection{Data extraction}

To retrieve quantitative article selection, two reviewers (AR and NV) independently screened the relevant titles and abstracts. After eligible articles inclusion, to determine the risk of bias, the full-texts of all included articles were also precisely screened by two reviewers (AR and NV), independently. Meanwhile, any discrepancies were arbitrated by a third reviewer (FS). Endnote X9 as a reference management software (Thomson Corporation Inc., USA) was used to organize titles and abstracts of studies as well as duplicated identification. It should be noted that corresponding authors of primary studies were contacted for any missing or clarifying unclear data, where required. Finally, required data extraction from the articles was summarized in the extraction diagrams (Table 1, Ref. [10, 14-21] and Table 2, Ref. [22-41]) and intended study design items including first author's name, year of publication, study location, type of animals (species, sex), sample size, name of therapeutic agents, related-dose, route of administration, experimental model of ischemic stroke, and infarct size alternation $\left(\%\right.$ or $\left.\mathrm{mm}^{3}\right)$ were prepared.

\subsection{Statistical meta-analysis}

The numbers of animals and average stroke volume (mean $\pm \mathrm{SD}$ ) in each group were extracted from the included articles. Next, the differentiation of the stroke volume for each study was calculated, and then the pooled mean differences were achieved by meta-analysis. To combine mean differences, the random effect model was used whereas the heterogeneity between studies was assessed by Cochran statistics (Q) and $\mathrm{I}^{2}$ test, which demonstrate the percentage of the variance between studies. For data analysis, CMA software was applied. To assess the publication bias, Egger's regression test and the Funnel Plot were used. Besides, to further evaluation of possible publication bias, the Trim and Fill method was performed. Effect sizes were also expressed as pool mean differences (for continuous data) and their 95\% confidence interval (CI) was calculated for further analysis. Regarding the subgroup analysis, it could be calculated when there is adequate data. Finally, these findings were presented in a description form to assist in data presentation where statistical pooling is not possible. The $p$-value of less than 0.05 was considered statistically significant.

\section{Results}

\subsection{Advanced search features}

Following the systemic search using the database, 3551 articles were identified. 2363 duplicated and 933 irrelevant articles were excluded after a preliminary evaluation of the articles according to the title and abstract. Following the fulltext assessment for article eligibility, of a total number of 256, 227 articles were also excluded. Ultimately, 29 articles supporting the inclusion criteria were included in the current meta-analysis. The relevant flow chart of determined and included articles was outlined in Appendix Fig. 5. According to the obtained data from the included articles, the animals were assigned to the control group without any intervention, the stroke group induced by permanent/transient MAOC manner, and treatment groups received autophagy modulators. 
A

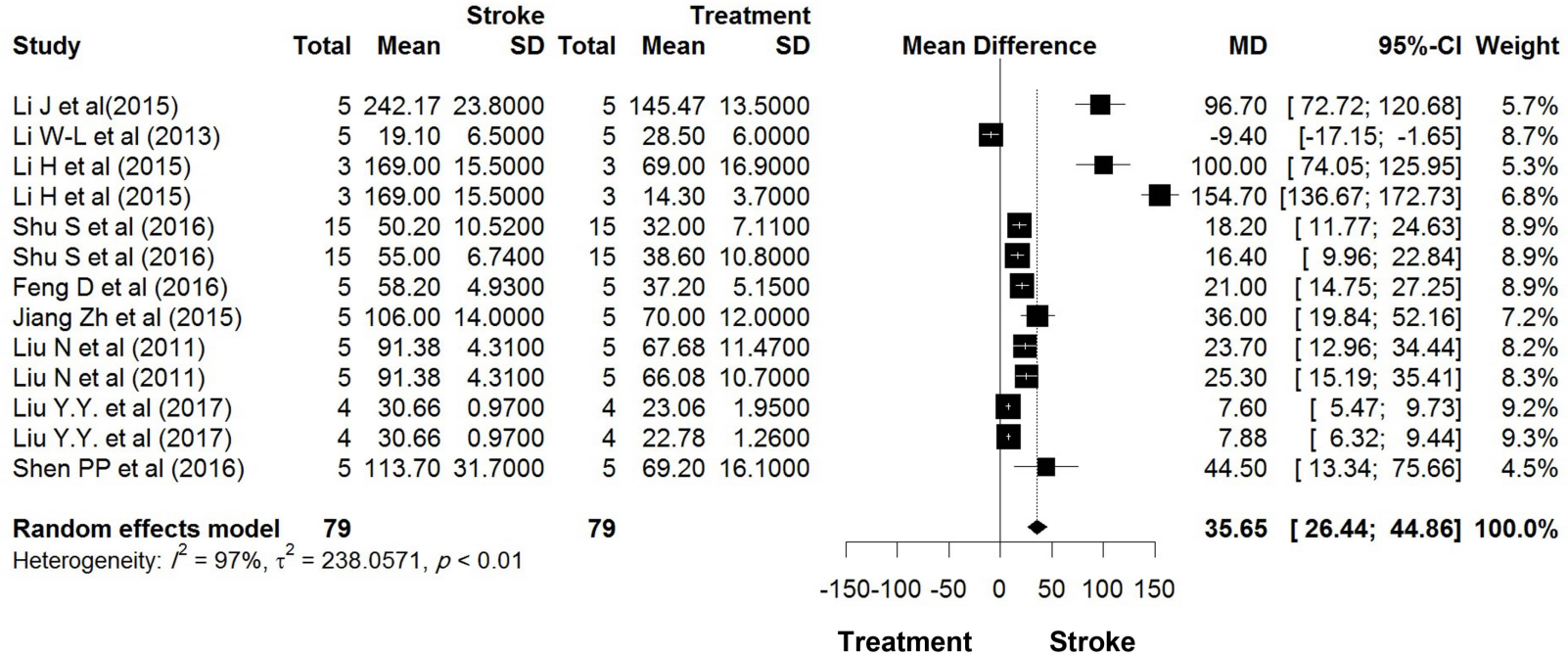

B

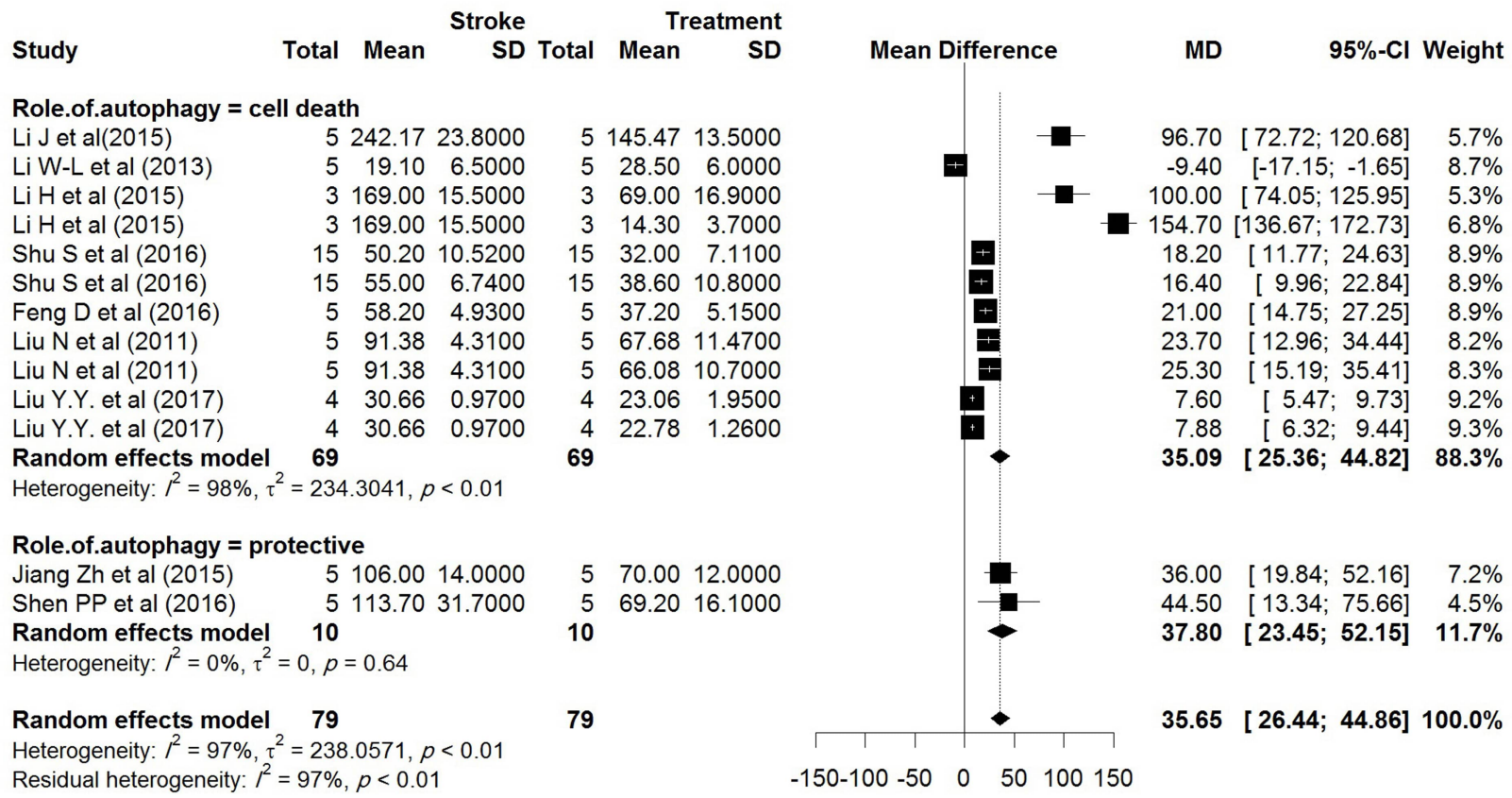

Treatment Stroke

Fig. 1. Effect of autophagy process on stroke volume based on $\mathbf{m m}^{3}$ measurement. (A) Forest panel analysis represented by mean differences and $95 \%$ CIs following the search strategy till 2021. (B) Subgroups analysis according to the cell death/protective role of the autophagy) represented by mean differences and $95 \%$ CIs, showing that the autophagy process mainly involves in the stroke volume progression and subsequently promotes the cell death. 
Table 1. Designed characteristics of included studies based on $\mathbf{~ m m}^{3}$.

\begin{tabular}{|c|c|c|c|c|c|c|c|c|c|c|}
\hline Publication & Year & Country & Species \& Gender & Sample size (n) & (Dose, route of delivery) & $\begin{array}{l}\text { Time course of } \\
\text { autophagy assessment (h) }\end{array}$ & $\begin{array}{c}\text { Levels of LC3 } \\
\text { after Treatment }\end{array}$ & Temp/Perm & Infarct $\left(\mathrm{mm}^{3}\right)$ & ) Outcome (autophagy effect) \\
\hline Li J et al. [14] & 2015 & China & Female SD rats & 5 & 17- AAG (80 mg/kg), i.p. & 24 & Decreased & Temp & $96.7 \pm 12.23$ & Cell death \\
\hline Li W-L et al. [15] & 2013 & USA & $\begin{array}{l}\text { Male wild-type (B6, 129PF2) } \\
\text { and p50 knockout (p50, B6, } \\
\text { 129P-Nfkb1) mice }\end{array}$ & 5 & NF-kB & 12,24 & Decreased & Perm & $-9.4 \pm 3.65$ & Cell death \\
\hline Li H et al. [16] & 2015 & China & Male SD rats & 3 & $002 \mathrm{C}-3(10 \mathrm{~g} / \mathrm{kg})$, i.v. & 24 & Decreased & Temp & $100 \pm 13.24$ & Cell death \\
\hline Li H et al. [16] & 2015 & China & Male SD rats & 3 & $002 \mathrm{C}-3(50 \mathrm{~g} / \mathrm{kg})$, i.v. & 24 & Decreased & Temp & $154.7 \pm 9.2$ & Cell death \\
\hline Shu S et al. [17] & 2016 & China & Male SD rats & 15 & EA $24 \mathrm{~h}$ & $6,24,72$ & Decreased & Temp & $18.2 \pm 3.27$ & Cell death \\
\hline Shu S et al. [17] & 2016 & China & Male SD rats & 15 & EA72 h & $6,24,72$ & Decreased & Temp & $16.4 \pm 3.2$ & Cell death \\
\hline Feng D et al. [10] & 2016 & China-USA & Male C57BL/6 mice & $\mathrm{KN}$ & $\operatorname{Mel}(10 \mathrm{mg} / \mathrm{kg})$, i.p. & $6,12,24$ & Decreased & Temp & $21.00 \pm 3.18$ & Cell death \\
\hline Liu N et al. [18] & 2011 & Japan & Male C57BL/6 mice & 5 & Edaravone $\mathrm{A}, 9 \mathrm{mg} / \mathrm{kg}$ i.v. & 48 & Decreased & Temp & $23.7 \pm 5.47$ & Cell death \\
\hline Liu N et al. [18] & 2011 & Japan & Male C57BL/6 mice & 5 & Edaravone B, 9 mg $/ \mathrm{kg}$ i.v. & 48 & Decreased & Temp & $25.3 \pm 5.15$ & Cell death \\
\hline Liu Y.Y. et al. [19] & 2017 & China & Male SD rats & 4 & PF11 (6, mg/kg), i.v. & 24 & Decreased & Perm & $7.6 \pm 1.09$ & Cell death \\
\hline Liu Y.Y. et al. [19] & 2017 & China & Male SD rats & 4 & $\operatorname{PF} 11$ (6, mg/kg), i.v. & 24 & Decreased & Perm & $7.88 \pm 0.79$ & Cell death \\
\hline Jiang Zh et al. [20] & $2015 \mathrm{C}$ & China and USA & Male SD rats & 5 & $\mathrm{MB}, 1$ mg/kg, i.p. & 24 & - & Temp & $36.00 \pm 8.24$ & Protective \\
\hline Shen PP et al. [21] & $2016 \mathrm{C}$ & China and USA & Male Wistar rats & 5 & CSD Preconditioning & $6,12,24$ & Increased & Temp & $10.62 \pm 1.5$ & Protective \\
\hline
\end{tabular}

17-AGG, 17-allylamino-17-demethoxygeldanamycin; CSD, Cortical Spreading Depression; MB, Methylene blue; Mel, Melatonin; NF- $\kappa$ B, Nuclear factor kappa B. 
Table 2. Designed characteristics of included studies and infarct size based on percentage (\%).

\begin{tabular}{|c|c|c|c|c|c|c|c|c|c|c|}
\hline Authors & Year & Country & Species \& Gender & Sample size (n) & $\begin{array}{c}\text { Dose \& route of delivery of } \\
\text { therapeutic agents }\end{array}$ & $\begin{array}{c}\text { Time course of } \\
\text { autophagy assessment (h) }\end{array}$ & $\begin{array}{c}\text { Level of LC3 after } \\
\text { Treatment }\end{array}$ & Temp/Perm & $\begin{array}{l}\text { Infarct size } \\
\text { reduction (\%) }\end{array}$ & $\begin{array}{c}\text { Outcome } \\
\text { (autophagy effect) }\end{array}$ \\
\hline Li Q et al. [22] & 2014 & China & Male wild-type ICR mice & $16-20$ & Rap $8 \mathrm{ng} / 2$ micro DMSO $0.1 \%$, i.c.v. & $6,24,48$, and 72 & Increased & Perm & $11.86 \pm 2.16$ & Protective \\
\hline $\mathrm{Bu} \mathrm{Q}$ et al. [23] & 2014 & China & MaleWild-type ICR mice + SD rats & 10 & w007B10 mg $/ \mathrm{kg}$, i.v. & 24 & Decreased & Temp & $16.8 \pm 1.44$ & Cell death \\
\hline Bu Q et al. [23] & 2014 & China & MaleWild-type ICR mice + SD rats & 10 & w007B 50 mg/kg, i.v. & 24 & Decreased & Temp & $35.7 \pm 1.16$ & Cell death \\
\hline Fu L et al. [24] & 2016 & China & Male Balb/c mice & 6 & CC (20 mg/kg), i.p. & 24 & Increased & Perm & $22.43 \pm 0.56$ & Cell death \\
\hline Li Y et al. [25] & 2015 & China & Male SD rats & 12 & Ebselen, gavage & 14 day & Decreased & Temp & $18.2 \pm 3.27$ & Cell death \\
\hline Chi O.Z. et al. [26] & 2016 & USA & Male Fischer Rat & 8 & Rap, 20 mg/kg, i.p. & 48 & Decreased & Temp & $16.4 \pm 3.2$ & Cell death \\
\hline Lu T et al. [27] & 2011 & China & Male SD rats & 3 & GRb1, $1.25 \mathrm{mg} / \mathrm{kg}$ intra nasal & 24 & Decreased & Temp & $23.14 \pm 1.23$ & Cell death \\
\hline Lu T et al. [27] & 2011 & China & Male SD rats & 3 & GRb1, $12.5 \mathrm{mg} / \mathrm{kg}$ intra nasal & 24 & Decreased & Temp & $29.81 \pm 1.13$ & Cell death \\
\hline Wu M et al. [28] & 2017 & China & Male SD rats & 6 & Pre- Rap (3.0 mg/kg.), i.p. & $24 \mathrm{~h}, 7$ days & Increased & Temp & $12.6 \pm 1.73$ & Protective \\
\hline Wu M et al. [28] & 2017 & China & Male SD rats & 6 & Post-Rap (3.0 mg/kg.), i.p. & $24 \mathrm{~h}, 7$ days & Increased & Temp & $8.3 \pm 1.46$ & Protective \\
\hline Qi Zh et al. [29] & 2012 & China & Male SD rats & 4 & IPOC 10 & 24 & Increased & Temp & $22.00 \pm 2.75$ & Protective \\
\hline Qi Zh et al. [29] & 2012 & China & Male SD rats & 4 & IPOC 30 & 24 & Increased & Temp & $18.00 \pm 2.4$ & Protective \\
\hline Qi Zh et al. [30] & \multicolumn{2}{|c|}{2015 China and USA } & Male SD rats & 4 & RIC & 24 & Increased & Perm & $10.62 \pm 1.5$ & Protective \\
\hline Wang R et al. [31] & 2014 & China & Male Wistar rats & 6 & Res $30 \mathrm{mg} / \mathrm{kg}$, i.p. & 24 & Increased & Temp & $9.29 \pm 3.97$ & Protective \\
\hline Jeong J.H. et al. [32] & 2016 & Korea & Male SD rats & 5 & IF & 24 & Increased & Temp & $38.64 \pm 0.98$ & Protective \\
\hline Li L et al. [33] & \multicolumn{2}{|c|}{2017 China and USA } & Male SD rats & 6 & GM1 25 mg/kg, i.p. & 24 & Decreased & Perm/Temp & $6.8 \pm 1.57$ & Cell death \\
\hline Li L et al. [33] & \multicolumn{2}{|c|}{2017 China and USA } & Male SD rats & 6 & GM1 50 mg/kg, i.p. & 24 & Decreased & Perm/Temp & $1.6 \pm 1.91$ & Cell death \\
\hline Lu K.M. et al. [34] & 2019 & China & Male SD rats & 3 & $\mathrm{HBO}$ & $3,6,12,24$, and 48 & Decreased & Perm & $5.7 \pm 0.016$ & Cell death \\
\hline Li G et al. [35] & 2012 & China & Male Sprague-Dawley (SD) rats & 5 & IPOC & 24 & Decreased & Temp & $17.48 \pm 1.59$ & Cell death \\
\hline Qi Zh E et al. [36] & \multicolumn{2}{|c|}{2014 China and USA } & Male SD rats & $3-4$ & HSYA ( 2 mg/kg), i.v. & 24,48, and 72 & Increased & Temp & $10.62 \pm 2.26$ & Protective \\
\hline Chen et al. [37] & 2020 & China & Male ICR Mice & 6 & TAT-SPK2 (1 mg/kg/day), i.p. & $1,3,6,12$, and 24 & Increased & Temp & $49.7 \pm 7.2$ & Protective \\
\hline Chen et al. [37] & 2020 & China & Male ICR Mice & 6 & TAT-SPK2 (2 mg/kg/day), i.p. & $1,3,6,12$, and 24 & Increased & Temp & $41.9 \pm 11.2$ & Protective \\
\hline Chen et al. [37] & 2020 & China & Male ICR Mice & 6 & TAT-SPK2 (4 mg/kg/day), i.p. & $1,3,6,12$, and 24 & Increased & Temp & $34.2 \pm 8.3$ & Protective \\
\hline Li et al. [38] & 2020 & USA & Male C57/BL6J mice & 6 & $28 \%(2.8 \mathrm{~g} / \mathrm{kg} / \mathrm{d})$ Ethanol, Gavage & 24 & Decreased & Temp & $-20 \%$ & Protective \\
\hline Pan et al. [39] & 2020 & China & Male Sprague-Dawley rats & 16 and 32 & Treadmill & 3 and 7 days & Decreases & Temp & $20.72 \pm 2.62$ & Cell death \\
\hline Wang et al. [40] & 2020 & China & Male C57/BL6J mice & 8 & STS, $10 \mathrm{mg} / \mathrm{kg}$, i.p. & 1 and 3 & Decreases & Temp & $29.81 \pm 3.35$ & Cell death \\
\hline Wang et al. [40] & 2020 & China & Male C57/BL6J mice & 8 & STS, $20 \mathrm{mg} / \mathrm{kg}$, i.p. & 1 and 3 & Decreases & Temp & $22.71 \pm 3.55$ & Cell death \\
\hline Wang et al. [40] & 2020 & China & Male C57/BL6J mice & 8 & STS, 40 mg/kg, i.p. & 1 and 3 & Decreases & Temp & $21.59 \pm 2.95$ & Cell death \\
\hline Wang et al. [41] & 2021 & China & Male Sprague-Dawley rats & 6 & $\begin{array}{l}\text { HBO } 100 \% \text { oxygen and } 1.5 \\
\text { atmosphere absolute pressure }\end{array}$ & 72 & Decreases & Temp & $20.12 \pm 2.940$ & Cell death \\
\hline
\end{tabular}

CC, C compound; EA, Electroacupuncture; GM,1 Ganglioside; HBO, Hyperbaric Oxygen Therapy; HSYA, Hydroxysafflor yellow A; IPOC, Ischemic Post conditioning; Mel, Melatonin; Rap, Rapamycin; Res, Resveratrol; STS, Sodium tanshinone IIA sulfonate; TAT-SPK2, Sphingosine Kinase 2-mimicking TAT-peptide. 


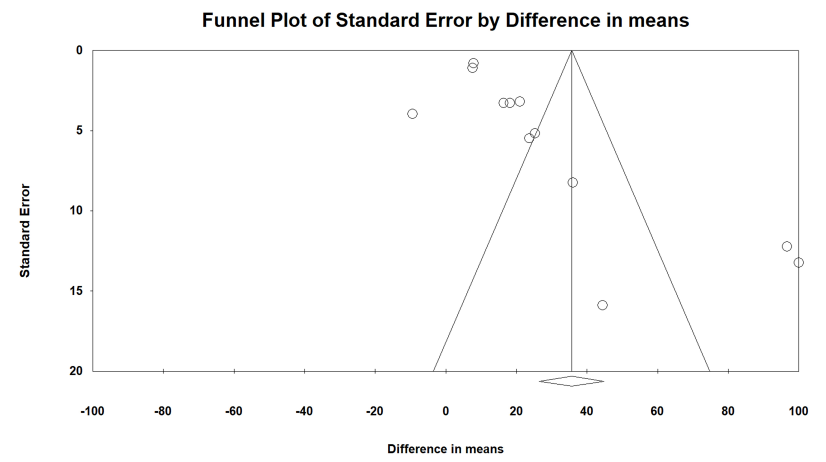

Fig. 2. Funnel plot of publication bias between studied groups calculated by egger's regression test. Pooled mean difference (CI: 95\%).

\subsection{Differences in mean of stroke volume based on $\mathrm{mm}^{3}$}

In 13 studies, the mean of stroke volume has been calculated while the heterogeneity between included studies was significant $\left(\mathrm{Q}\right.$-value $=59.83, \mathrm{df}=12, p<0.001, \mathrm{I}^{2}=$ $79.94 \%)$. According to the meta-analysis results, the pooled mean difference of stroke volume between stroke and treatment groups was 35.65 units $(\mathrm{MD}=35.65,95 \% \mathrm{CI}=26.43$ to 44.85 , z-value $=7.58, p<0.001$ ). In Fig. $1 \mathrm{~A}$, the forest plot analysis showed that autophagy activation in 11 and 2 studies contributed to cell death and protection, respectively. The Forest plot of the subgroup analysis was also shown in Fig. 1B. Based on the obtained results, in studies that reported the autophagy negative effect $(n=11)$, the results of subgroup analysis showed that pooled mean difference in terms of stroke volume between stroke and treatment groups was 35.06 units $(\mathrm{MD}=35.06,95 \% \mathrm{CI}=25.35$ to 44.77 , $\mathrm{z}$-value $=7.08, p<0.001)$. Additionally, in studies with the protective role of autophagy $(n=2)$, the pooled mean difference of stroke volume between the two groups was estimated 39.25 units $(\mathrm{MD}=39.25,95 \% \mathrm{CI}=12.53$ to $65.97, \mathrm{z}$-value $=2.88, p$ $<0.001)$.

\subsubsection{Publication bias}

The relevant publication bias for the funnel plot has been shown in Fig. 2. According to the consequence of the stroke volume mean difference, egger's regression test revealed that publication bias was practically significant between studied groups $(\mathrm{t}$-value $=3.24, \mathrm{df}=11, p$-value $=0.007)$. Moreover, the Trim and Fill method was performed for publication bias modifying, which added one study for missed study modulation. The results of this analysis also showed that the adjusted pooled mean difference for stroke volume between the two groups was 39.25 units $(\mathrm{AMD}=41.92,95 \% \mathrm{CI}=30.33$ to 53.51).

\subsubsection{Sensitivity analysis}

According to the results shown in Fig. 1, studies conducted by Li J et al. [14], and Li H et al. [16], could be considered as a source of heterogeneity among studies. Thereby, the sensitivity analysis was performed regardless of these studies.
Based on the results of sensitivity analysis, it has been clarified that pooled mean difference for stroke volume between stroke and treatment groups was $15.09(\mathrm{MD}=15.09,95 \% \mathrm{CI}$ : 10.12 to 20.04 , z-value $=5.95$, $p$-value $<0.001$ ), while for studies with the detrimental effect of autophagy the sensitivity analysis was estimated $12.98(\mathrm{MD}=12.98,95 \% \mathrm{CI}$ : 8.21 to 17.75. $\mathrm{z}$-value $=5.33, p$-value $<0.001)$.

\subsection{Differences in mean of stroke volume based on the percentage (\%)}

Based on the percentage of the infarct volume mean, which has been reported in 29 studies, the heterogeneity between the studies was also statistically significant $(\mathrm{Q}$-value $=$ 4830.82, $\mathrm{df}=28, p<0.001, \mathrm{I}^{2}=99.40 \%$ ). In Fig. $3 \mathrm{~A}$, the forest plot of combined results has been depicted in detail, which showed that the pooled mean difference for stroke volume between stroke and treatment groups was14.38\% (MD $=14.38,95 \% \mathrm{CI}=10.50$ to $18.26, \mathrm{z}$-value $=7.26, p<0.001$ ) (Fig. 3A). The related forest plot of subgroup analysis has been summarized in Fig. 3B. As shown in Fig. 3B, autophagy exhibited a cell death effect in 17 studies while 12 studies reported the protective role of autophagy. In this respect, the subgroup analysis by considering the autophagy controversy effects showed that the pooled mean difference for stroke volume between stroke and treatment groups regarding the cell death outcome was $12.52 \%(\mathrm{MD}=12.52$, 95\% CI: 7.91 to 17.14 , $\mathrm{z}$-value $=5.33, p<0.001)$. In addition, the studies in which the protective role of autophagy towards the infarct volume progression were proved indicated that the pooled mean difference for stroke volume between two groups was $17.12 \%(\mathrm{MD}=17.12$, $95 \% \mathrm{CI}: 9.08$ to 25.15 , $\mathrm{z}$-value $=4.18, p$ $<0.001)$

\section{Publication bias}

Publication bias assessment of the mean differences of the stroke volume has been shown in the funnel plot (Fig. 4). According to egger's regression test, there was no significant publication bias between different groups $(\mathrm{t}$-value $=1.96 \mathrm{df}$ $=27, p=0.06$ ).

\section{Discussion}

To the best of our knowledge, IS, as a more common type of stroke and a devastating disease, is mainly characterized by the major lack of regional cerebral blood supply in a distinct area of the cerebral tissue [42]. IS could be defined as one of the major leading causes of a corresponding loss of neurologic function, particularly in the aging population [43, 44]. Besides the dysregulated autophagy, it has been also documented that other pathological conditions such as mitochondrial dysfunction, oxidative stress, acidosis, calcium overload, and inflammatory response are associated with the pathogenesis of cerebral ischemia-reperfusion injury (IRI) [45]. The current systematic review and meta-analysis aimed to clarify autophagy modulation (either inhibition or induction) and its possible effects on the histological and infracted volume restoration in animal models of ischemic stroke. As men- 
A

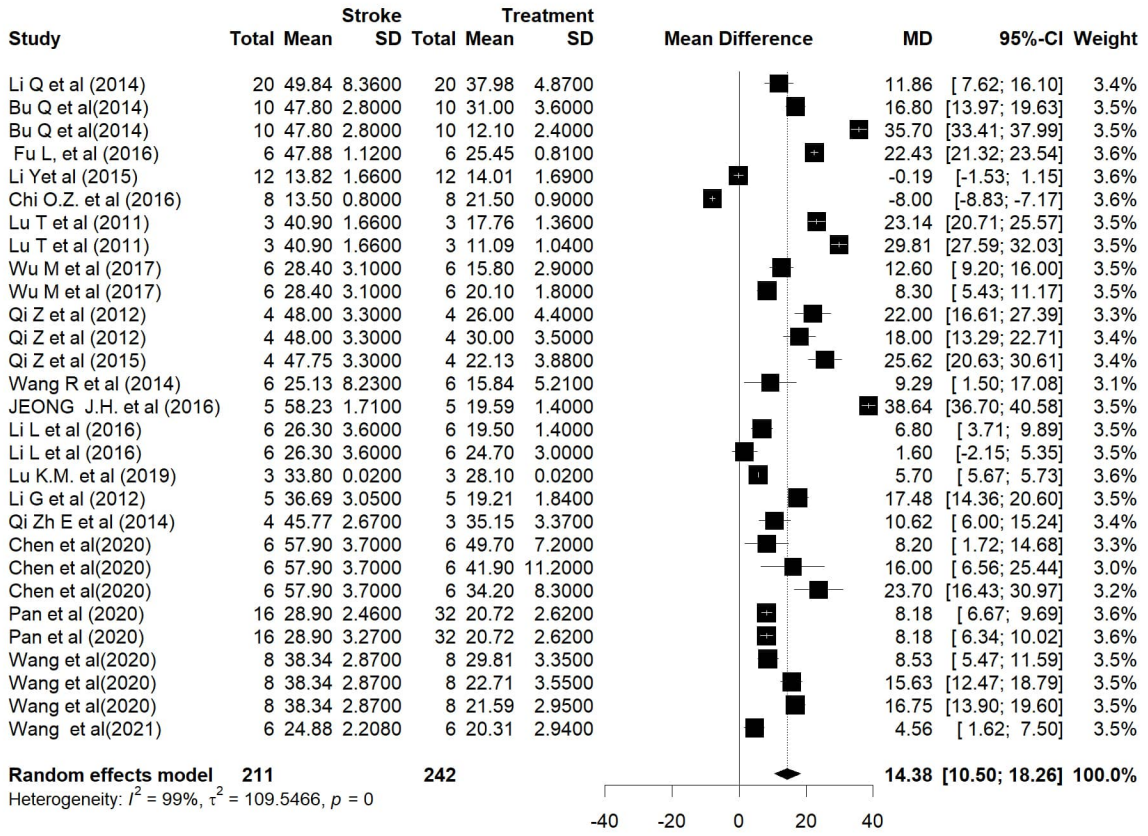

B

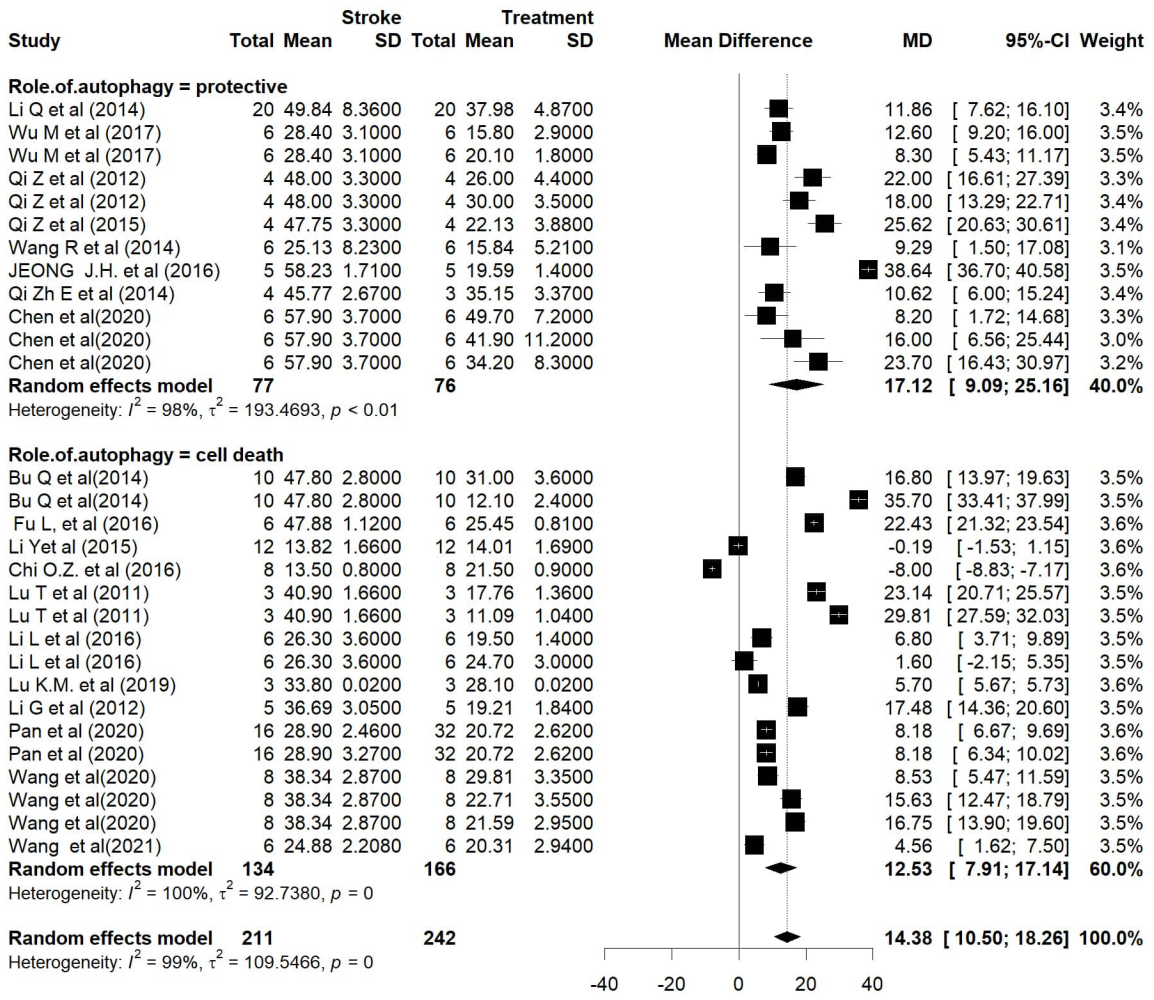

Fig. 3. Effect of autophagy modulation on stroke volume based on percentage (\%). (A) Forest panel analysis according to included studies and represented by mean differences and 95\% CIs, following the search strategy till 2021, (B) Subgroups analysis according to the cell death/protective role of the autophagy represented by mean differences and 95\% CIs, showing that the autophagy process mainly involves in the stroke volume progression and subsequently promotes the cell death. 


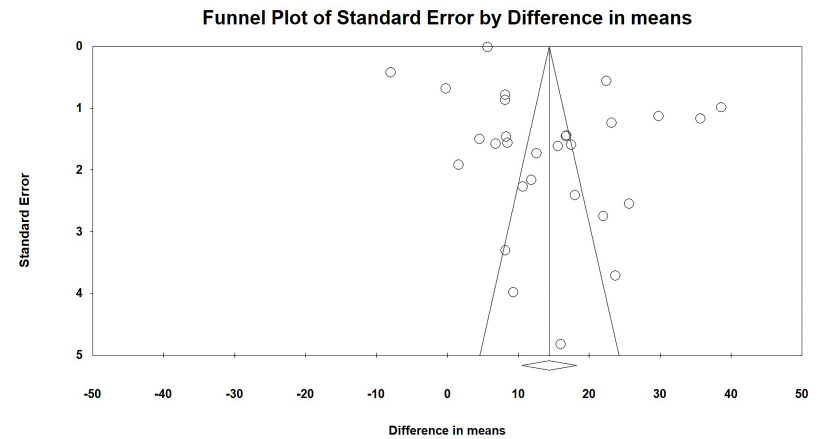

Fig. 4. Funnel plot of publication bias between studied groups calculated by egger's regression test. Pooled mean difference (CI: 95\%).

tioned earlier, the basal level of autophagy is considered as an obligatory factor for neuronal normal activity while autophagy dysregulation promotes neurodegeneration, as well as misfolded protein aggregation [46]. Moreover, mounting evidence highlighted the causal role of autophagy activity during IS [47-49]. In detail, recent publications indicated that following the acute and severe IS, autophagic subtypes including mitophagy, pexophagy, lipophagy, and endoplasmic reticulophagy are predominantly involved in IS progression [50]. To further establish this finding, using transmission electron microscopy represented the increased amount of autophagosomes, named bilayer-membrane autophagic vacuoles, in the damaged ischemic neurons, which further highlighted the autophagy involvement in cerebral pathology induced by IS [51]. In this line, our results pointed out that autophagy efficacy predominately emerges in a time course between 6-72 $\mathrm{h}$ in terms of both cell protection and cell death status. Meanwhile, other variables such as gender, different anesthetic drugs used, route of administration, and different procedures for stroke induction had no significant bearing on autophagy consequences. Even so, the clinical application of autophagy modulators in diagnosed stroke patients is still restricted due to the plenty of contradictory studies. Given the limited studies conducted in this era, it could be assumed that there is a high risk of bias and suggesting further pre-clinical studies to confirm the exact role of autophagy in terms of the stroke volume modification with considering time-dependent effectiveness; However, to further establish of these findings, a comprehensive estimation of 29 studies, in which determined the infarct size using either percentage (\%) or ischemic area measurement $\left(\mathrm{mm}^{3}\right)$ revealed that 17 and 12 articles through the autophagy cessation and stimulation gained the parallel results and markedly decreased infarct size by $15.41 \%$ and $35.65 \mathrm{~mm}^{3}$, respectively. According to the recent systematic review, rapamycin (Sirolimus), an immunosuppressive drug that induces moderate autophagy by inhibition of the mammalian target of rapamycin (mTOR), exhibited a great beneficial effect for infarct volume reduction and ongoing neuroprotection effect, particularly in lower doses (8 ng) $[52,53]$. Similarly, our analysis also demonstrated that the neuroprotective ef- fects of different agents or conditions mostly mediated by autophagy inhibition resulted in infracted volume reduction. This outcome strongly implicated that prolonged stimulation, as well as the overexpression of autophagy, plays a major role in infarct size progression in stroke subjects, which negatively could exhibit in the high level of rapamycin $(20$ $\mathrm{mg} / \mathrm{kg}$ ), as well. In a study conducted by Chi et al. [26], it has been shown that mTOR, as a main target of rapamycin, exerts an imperative role not only in the maintenance of the cellular survival also governs the oxygen balance following the cerebral IRI likely through AKT and S6K1 phosphorylation in the cerebral cortex. Therefore, the high dose of rapamycin can increase infarct volume via $\mathrm{mTOR}$ inhibition as well as limitation of $\mathrm{O}_{2}$ consumption during reperfusion [26]. Notably, the protective effects in neural cells induced by autophagy, are predominately mediated using mTOR 1 inhibitors such as rapamycin and metformin preconditioning as well as mTOR2 activation [54]. While utilizing rapamycin in low doses may also have enhanced autophagy activity enough in a non-mTORC2 manner to maintain neuronal survival following ischemia. Another protective activity of autophagy intercedes by scavenging accumulated misfolded proteins and cytoplasmic worn-out components in response to acute IS $[55,56]$. To interrogate the exact role of the multi-phase autophagy process, a primary clinical trial to clarify the autophagy inhibitory/induction effect on mTOR2 is highly recommended in the context of IS. Another critical issue refers to the MCAO-induced IS leading to neuronal death by autophagosome accumulation and blocks autophagy flux in which increases the intracellular LC3, Beclin-1 (wellknown autophagic biomarkers), and P62 (an adaptor protein) that conversely shows autophagy flux $[19,57]$. The effect size of both autophagy modulations for infarct volume reduction was approximately equal. Together, there is no significant publication bias regarding the mean infarct volume percentage while publication bias was observed in mean differences of infarction volume amount $\left(\mathrm{mm}^{3}\right)$ between studied groups.

Regarding the latent underlying mechanisms of action involved in autophagy regulation, Zhang et al. [58] indicated that chloride channel-3, as a signal molecule, exerted a neuroprotective role, which can directly activate autophagy machinery through the interaction between Beclin1 and Vps34 in a self-protective manner to impede infarct volume progression following acute IS (AIS), in vivo. In contrast, it has been reported that FK506 binding protein 5 (FKBP5), as a novel prognostic and diagnostic value, is upregulated in subjects with AIS and participates in disease severity. FKBP5 by autophagy induction through the downstream AKT/FOXO3 blocking could promote AIS exacerbation [59]. Another target signaling pathway to suppress dysregulated autophagy refers to the AKT/mTOR axis stimuli as well as autophagy-related gene 7 ( Atg 7$)$ downregulation emerging by dichloromethane therapy against IS in rats [60]. Notably, the results of a recent study conducted by Cai et al. [61] also showed that one of the substantial mechanisms in- 
Table 3. MEDLINE was used to preliminary search strategy for autophgay and stroke.

\#15 Search $(()((()((()((()(()((()((()(((“ B i o m a r k e r s ”[M e s h]))$ OR Biologic Markers[Title]) OR Biologic Markers[Title/Abstract]) OR Serum

Marker[Title]) OR Serum Marker[Title/Abstract]) OR Endpoints, Surrogate[Title]) OR Endpoints, Surrogate[Title/Abstract]))) OR LC3II[Title/Abstract]) OR LC3I[Title/Abstract]) OR P62[Title/Abstract]) OR Beclin-1[Title/Abstract])) OR 'sequestosome 1'[Title/Abstract]) OR 'sqstm1 protein'[Title/Abstract]) OR 'protein sqstm1'[Title/Abstract]) OR 'protein p 62'[Title/Abstract]) OR 'protein p62'[Title/Abstract]) OR 'sequestosome-1 protein'[Title/Abstract]) OR 'ubiquitin binding protein p62'[Title/Abstract]) OR 'beclin 1'[Title/Abstract]) OR 'atg6 protein'[Title/Abstract]) OR 'becn1 protein'[Title/Abstract]) OR 'vps30 protein'[Title/Abstract]) OR 'coiled coil myosin like bcl2 interacting protein'[Title/Abstract]) OR ('protein atg6'[Title/Abstract] OR 'protein becn1'[Title/Abstract])) OR ('protein vps30'[Title/Abstract] OR 'protein beclin 1'[Title/Abstract])) OR 'protein beclin1'[Title/Abstract])))

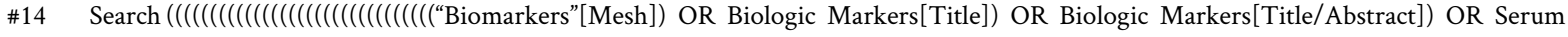
Marker[Title]) OR Serum Marker[Title/Abstract]) OR Endpoints, Surrogate[Title]) OR Endpoints, Surrogate[Title/Abstract]))) OR LC3II[Title/Abstract]) OR LC3I[Title/Abstract]) OR P62[Title/Abstract]) OR Beclin-1[Title/Abstract])) OR 'sequestosome 1'[Title/Abstract]) OR 'sqstm1 protein'[Title/Abstract]) OR 'protein sqstm1'[Title/Abstract]) OR 'protein p 62'[Title/Abstract]) OR 'protein p62'[Title/Abstract]) OR 'sequestosome-1 protein'[Title/Abstract]) OR 'ubiquitin binding protein p62'[Title/Abstract]) OR 'beclin 1'[Title/Abstract]) OR 'atg6 protein'[Title/Abstract]) OR 'becn1 protein'[Title/Abstract]) OR 'vps30 protein'[Title/Abstract]) OR 'coiled coil myosin like bcl2 interacting protein'[Title/Abstract]) OR ('protein atg6'[Title/Abstract] OR 'protein becn1'[Title/Abstract])) OR ('protein vps30'[Title/Abstract] OR 'protein beclin 1'[Title/Abstract])) OR 'protein beclin1'[Title/Abstract])) AND (((((()(((“Autophagy”[Mesh]) OR Autophag*[Title]) OR Autophag*[Title/Abstract]) OR Macro autophag*[Title]) OR Macro autophag*[Title/Abstract]) OR Autophag* Cellular[Title]) OR Autophag* Cellular[Title/Abstract]) OR Programmed Cell Death, Type II[Title]) OR Programmed Cell

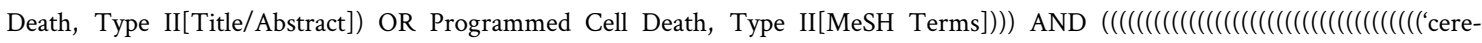
brovascular accident'[MeSH Subheading]) OR CVA[Title/Abstract]) OR 'cerebrovascular accident'[Title/Abstract]) OR 'accident, cerebrovascular'[Title/Abstract]) OR 'acute cerebrovascular lesion'[Title/Abstract]) OR 'acute focal cerebral vasculopathy'[Title/Abstract]) OR 'acute stroke'[Title/Abstract]) OR 'apoplectic stroke'[Title/Abstract]) OR apoplex*[Title/Abstract]) OR 'blood flow disturbance, brain'[Title/Abstract]) OR 'brain accident'[Title/Abstract]) OR 'brain attack'[Title/Abstract]) OR 'brain blood flow disturbance'[Title/Abstract]) OR 'brain insult'[Title/Abstract]) OR 'brain insultus'[Title/Abstract]) OR 'brain ischaemic attack'[Title/Abstract]) OR 'brain ischemic attack'[Title/Abstract]) OR 'brain vascular accident' [Title/Abstract]) OR 'cerebral apoplexia'[Title/Abstract]) OR 'cerebral insult'[Title/Abstract]) OR 'cerebral stroke'[Title/Abstract]) OR 'cerebral vascular accident'[Title/Abstract]) OR 'cerebral vascular insufficiency'[Title/Abstract]) OR 'cerebro vascular accident'[Title/Abstract]) OR 'cerebrovascular accident'[Title/Abstract]) OR 'cerebrovascular arrest'[Title/Abstract]) OR 'cerebrovascular failure'[Title/Abstract]) OR 'cerebrovascular injury'[Title/Abstract]) OR 'cerebrovascular insufficiency'[Title/Abstract]) OR 'cerebrovascular insult'[Title/Abstract]) OR 'cerebrum vascular accident'[Title/Abstract]) OR 'cryptogenic stroke'[Title/Abstract]) OR 'ischaemic cerebral attack'[Title/Abstract]) OR 'ischaemic seizure'[Title/Abstract]) OR 'ischemic cerebral attack'[Title/Abstract]) OR 'ischemic seizure'[Title/Abstract]) OR stroke[Title/Abstract])

\#11 Search ((((()((((“Biomarkers”[Mesh]) OR Biologic Markers[Title]) OR Biologic Markers[Title/Abstract]) OR Serum Marker[Title]) OR Serum Marker[Title/Abstract]) OR Endpoints, Surrogate[Title]) OR Endpoints, Surrogate[Title/Abstract]))) OR LC3II[Title/Abstract]) OR LC3I[Title/Abstract]) OR P62[Title/Abstract]) OR Beclin-1[Title/Abstract]

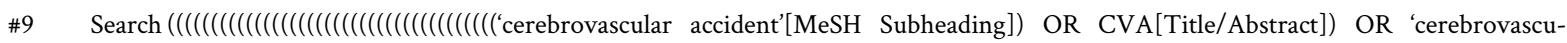
lar accident'[Title/Abstract]) OR 'accident, cerebrovascular'[Title/Abstract]) OR 'acute cerebrovascular lesion'[Title/Abstract]) OR 'acute focal cerebral vasculopathy'[Title/Abstract]) OR 'acute stroke'[Title/Abstract]) OR 'apoplectic stroke'[Title/Abstract]) OR apoplex*[Title/Abstract]) OR 'blood flow disturbance, brain'[Title/Abstract]) OR brain accident'[Title/Abstract]) OR brain attack'[Title/Abstract]) OR 'brain blood flow disturbance'[Title/Abstract]) OR 'brain insult'[Title/Abstract]) OR 'brain insultus'[Title/Abstract]) OR 'brain ischaemic attack'[Title/Abstract]) OR 'brain ischemic attack'[Title/Abstract]) OR 'brain vascular accident'[Title/Abstract]) OR 'cerebral apoplexia'[Title/Abstract]) OR 'cerebral insult'[Title/Abstract]) OR 'cerebral stroke'[Title/Abstract]) OR 'cerebral vascular accident'[Title/Abstract]) OR 'cerebral vascular insufficiency'[Title/Abstract]) OR 'cerebro vascular accident'[Title/Abstract]) OR 'cerebrovascular accident'[Title/Abstract]) OR 'cerebrovascular arrest'[Title/Abstract]) OR 'cerebrovascular failure'[Title/Abstract]) OR 'cerebrovascular injury'[Title/Abstract]) OR 'cerebrovascular insufficiency'[Title/Abstract]) OR 'cerebrovascular insult'[Title/Abstract]) OR 'cerebrum vascular accident'[Title/Abstract]) OR 'cryptogenic stroke'[Title/Abstract]) OR 'ischaemic cerebral attack'[Title/Abstract]) OR 'ischaemic seizure'[Title/Abstract]) OR 'ischemic cerebral attack'[Title/Abstract]) OR 'ischemic seizure'[Title/Abstract]) OR stroke[Title/Abstract])) AND (((((()(((“Autophagy”[Mesh]) OR Autophag*[Title]) OR Autophag*[Title/Abstract]) OR Macro autophag*[Title]) OR Macro autophag*[Title/Abstract]) OR Autophag* Cellular[Title]) OR Autophag* Cellular[Title/Abstract]) OR Programmed Cell Death, Type II[Title]) OR Programmed Cell Death, Type II[Title/Abstract]) OR Programmed Cell Death, Type II[MeSH Terms] )) 
Table 3. Continued.

Search Query Items found

\#6 Search $((((()(((((“$ Autophagy”[Mesh]) OR Autophag*[Title]) OR Autophag*[Title/Abstract]) OR Macro autophag*[Title]) OR Macro 716305 autophag*[Title/Abstract]) OR Autophag* Cellular[Title]) OR Autophag* Cellular[Title/Abstract]) OR Programmed Cell Death, Type II[Title]) OR Programmed Cell Death, Type II[Title/Abstract]) OR Programmed Cell Death, Type II[MeSH Terms]))) OR (((((((“Biomarkers”[Mesh]) OR Biologic Markers[Title]) OR Biologic Markers[Title/Abstract]) OR Serum Marker[Title]) OR Serum Marker[Title/Abstract]) OR Endpoints, Surrogate[Title]) OR Endpoints, Surrogate[Title/Abstract]))

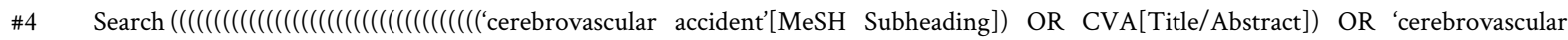
accident'[Title/Abstract]) OR 'accident, cerebrovascular'[Title/Abstract]) OR 'acute cerebrovascular lesion'[Title/Abstract]) OR 'acute focal cerebral vasculopathy'[Title/Abstract]) OR 'acute stroke'[Title/Abstract]) OR 'apoplectic stroke'[Title/Abstract]) OR apoplex*[Title/Abstract]) OR 'blood flow disturbance, brain'[Title/Abstract]) OR 'brain accident'[Title/Abstract]) OR brain attack'[Title/Abstract]) OR 'brain blood flow disturbance'[Title/Abstract]) OR 'brain insult'[Title/Abstract]) OR 'brain insultus'[Title/Abstract]) OR 'brain ischaemic attack'[Title/Abstract]) OR 'brain ischemic attack'[Title/Abstract]) OR 'brain vascular accident'[Title/Abstract]) OR 'cerebral apoplexia'[Title/Abstract]) OR 'cerebral insult'[Title/Abstract]) OR 'cerebral stroke'[Title/Abstract]) OR 'cerebral vascular accident'[Title/Abstract]) OR 'cerebral vascular insufficiency'[Title/Abstract]) OR 'cerebro vascular accident'[Title/Abstract]) OR 'cerebrovascular accident'[Title/Abstract]) OR 'cerebrovascular arrest'[Title/Abstract]) OR 'cerebrovascular failure'[Title/Abstract]) OR 'cerebrovascular injury'[Title/Abstract]) OR 'cerebrovascular insufficiency'[Title/Abstract]) OR 'cerebrovascular insult'[Title/Abstract]) OR 'cerebrum vascular accident'[Title/Abstract]) OR 'cryptogenic stroke'[Title/Abstract]) OR 'ischaemic cerebral attack'[Title/Abstract]) OR 'ischaemic seizure'[Title/Abstract]) OR 'ischemic cerebral attack'[Title/Abstract]) OR 'ischemic seizure'[Title/Abstract]) OR stroke[Title/Abstract]

\#3 Search (((c((“Biomarkers”[Mesh]) OR Biologic Markers[Title]) OR Biologic Markers[Title/Abstract]) OR Serum Marker[Title]) OR Serum Marker[Title/Abstract]) OR Endpoints, Surrogate[Title]) OR Endpoints, Surrogate[Title/Abstract])

\#2 Search $((((()(((()(“ S t r o k e ”[M e s h]))$ OR Stroke[Title]) OR Stroke[Title/Abstract]) OR Cerebrovascular Disorders[Title]) OR Cerebrovascular Disorders[Title/Abstract]) OR Cerebrovascular Accident[Title]) OR Cerebrovascular Accident[Title/Abstract]) OR CVA[Title]) OR CVA[Title/Abstract]) OR Cerebrovascular Apoplexy[Title]) OR Cerebrovascular Apoplexy[Title/Abstract]) OR Cerebral Stroke[Title]) OR Cerebral Stroke[Title/Abstract])

\#1 Search $(((((()(($ “Autophagy”[Mesh]) OR Autophag*[Title]) OR Autophag*[Title/Abstract]) OR Macro autophag*[Title]) OR Macro autophag*[Title/Abstract]) OR Autophag* Cellular[Title]) OR Autophag* Cellular[Title/Abstract]) OR Programmed Cell Death, Type II[Title]) OR Programmed Cell Death, Type II[Title/Abstract]) OR Programmed Cell Death, Type II[MeSH Terms])

volved in the neuroprotective role of tissue-type plasminogen activator (tPA), a well-known thrombolytic medication in the clinical treatment of cerebral IRI, e.g., IS, is mainly related to the activation of FUN14 domain-containing 1 (FUNDC1)-mediated mitophagy to retrieve mitochondrial dysfunction following the AMPK phosphorylation and subsequent apoptotic cell reduction. Previously, it has been reported that the elevated level of inflammatory mediators, such as annexin A1 and monomeric C-reactive protein, can worsen the prognosis of the post-ischemic aged brain, in vivo $[62,63]$. Interestingly, a cross-talk between autophagy and inflammation has also been delineated, which corroborated the benefits of moderate autophagy in facing post-stroke inflammatory response through the mTOR/AMPK pathway and subsequent inflammasome inhibitions [64]. Collectively, beyond the existing conventional therapies, novel therapeutic approaches such as hypothermia-induced infarct size reduction and autophagy modulation are of great significance, recently [50, 62]. Even so, as a limitation of the current study, the possible effect of some critical risk factors including aging, co-morbidities, and raised inflammatory mediators should be considered in upcoming studies, as well.

\section{Conclusions}

Given the conflict effects of autophagy regarding the infarct volume reduction, the studies included in this metaanalysis mostly reported a negative relation between autophagy induction and stroke volume development due to excessive autophagy activity following severe IS; in hence, it seems that further studies are also required to explore the underlying mechanisms to clarify the exact intervention role of autophagy modulation during cerebral ischemia for translating the potential therapeutic target in stroke patients.

\section{Abbreviations}

AIS, Acute Ischemic stroke; CI, confidence intervals; CMA, Comprehensive Meta-analysis; FKBP5, FK506 binding protein 5; IS, Ischemic stroke; IRI, ischemia-reperfusion injury; LC3, microtubule-associated protein 1A light chain 3; MCAO, middle cerebral artery occlusion; mTOR, mammalian target of rapamycin; SD, standard deviation.

\section{Author contributions}

AR-Designed the study; NV and FS-Performed search strategy; HH-Performed the methodological analysis; RRRevised the final draft; YS-Contributed to writing the manuscript; SS-Interpreted the analyzed Data. 


\section{Ethics approval and consent to participate}

The ethic number approved by Ethics Committee of Tabriz University of Medical Sciences for this study is IR.TBZMED.REC.1398.294.

\section{Acknowledgment}

Not applicable.

\section{Funding}

This review was supported by the Aging Research Institute of the Tabriz University of Medical Science with code number: 62827.

\section{Conflict of interest}

The authors declared no conflict of interest.

\section{Appendix}

See Table 3, Fig. 5.
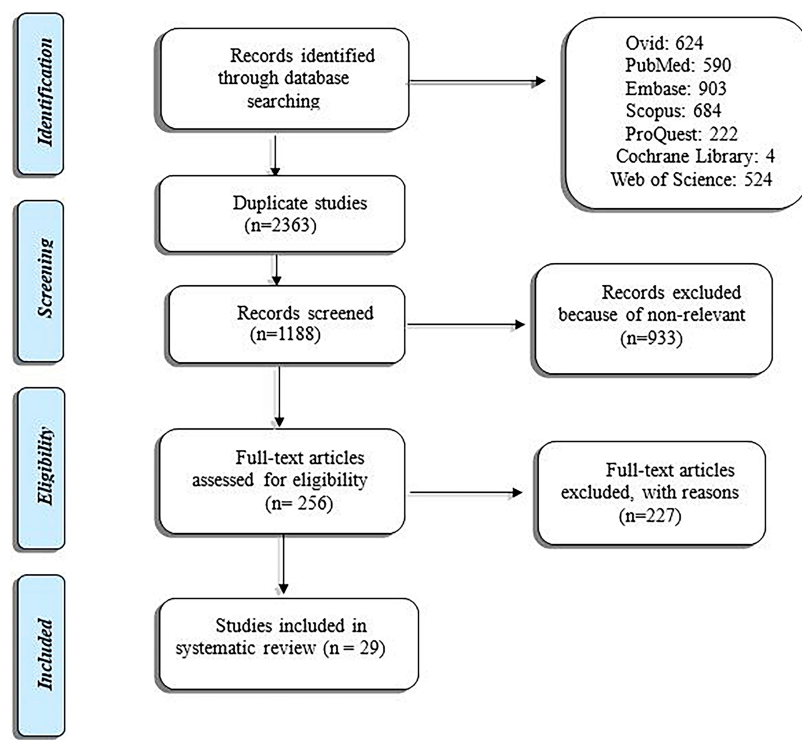

Fig. 5. Search and selection process of systematic review.

\section{References}

[1] Katan M, Luft A. Global burden of stroke. Seminars in neurology. 2018; 38: 208-211.

[2] Li L, Tian J, Long MK, Chen Y, Lu J, Zhou C, et al. Protection against Experimental Stroke by Ganglioside GM1 is Associated with the Inhibition of Autophagy. PLoS ONE. 2016; 11: e0144219.

[3] Yamal J, Rajan SS, Parker SA, Jacob AP, Gonzalez MO, Gonzales $\mathrm{NR}$, et al. Benefits of stroke treatment delivered using a mobile stroke unit trial. International Journal of Stroke. 2018; 13: 321327.

[4] Kokaia Z, Darsalia V. Human neural stem cells for ischemic stroke treatment. Results and Problems in Cell Differentiation. 2018; 66: 249-263.

[5] Ginet V, Spiehlmann A, Rummel C, Rudinskiy N, Grishchuk $\mathrm{Y}$, Luthi-Carter R, et al. Involvement of autophagy in hypoxicexcitotoxic neuronal death. Autophagy. 2014; 10: 846-860.

[6] Rezabakhsh A, Rahbarghazi R, Malekinejad H, Fathi F, Montaseri A, Garjani A. Quercetin alleviates high glucose-induced dam- age on human umbilical vein endothelial cells by promoting autophagy. Phytomedicine. 2019; 56: 183-193.

[7] Rezabakhsh A, Ahmadi M, Khaksar M, Montaseri A, Malekinejad $\mathrm{H}$, Rahbarghazi R, et al. Rapamycin inhibits oxidative/nitrosative stress and enhances angiogenesis in high glucose-treated human umbilical vein endothelial cells: Role of autophagy. Biomedicine \& Pharmacotherapy. 2017; 93: 885-894.

[8] Kroemer G. Autophagy: a druggable process that is deregulated in aging and human disease. The Journal of Clinical Investigation. 2015; 125: 1-4.

[9] Rezabakhsh A, Fathi F, Bagheri HS, Malekinejad H, Montaseri A, Rahbarghazi R, et al. Silibinin protects human endothelial cells from high glucose-induced injury by enhancing autophagic response. Journal of Cellular Biochemistry. 2018; 119: 8084-8094.

[10] Feng D, Wang B, Wang L, Abraham N, Tao K, Huang L, et al. Pre-ischemia melatonin treatment alleviated acute neuronal injury after ischemic stroke by inhibiting endoplasmic reticulum stressdependent autophagy via PERK and IRE1 signalings. Journal of Pineal Research. 2017; 62: e12395.

[11] Luo C, Ouyang M, Fang Y, Li S, Zhou Q, Fan J, et al. Dexmedetomidine Protects Mouse Brain from Ischemia-Reperfusion Injury via Inhibiting Neuronal Autophagy through up-Regulating HIF$1 \alpha$. Frontiers in Cellular Neuroscience. 2017; 11: 197.

[12] Puyal J, Clarke PGH. Targeting autophagy to prevent neonatal stroke damage. Autophagy. 2009; 5: 1060-1061.

[13] Sheng R, Liu X, Zhang L, Gao B, Han R, Wu Y, et al. Autophagy regulates endoplasmic reticulum stress in ischemic preconditioning. Autophagy. 2012; 8: 310-325.

[14] Li J, Yang F, Guo J, Zhang R, Xing X, Qin X. 17-AAG posttreatment ameliorates memory impairment and hippocampal CA1 neuronal autophagic death induced by transient global cerebral ischemia. Brain Research. 2015; 1610: 80-88.

[15] Li W-L, Yu SP, Chen D, Yu SS, Jiang Y-, Genetta T, et al. The regulatory role of NF- $\mathrm{xB}$ in autophagy-like cell death after focal cerebral ischemia in mice. Neuroscience. 2013; 244: 16-30.

[16] Li H, Liu X, Zhu Y, Liu Y, Wang Y. Magnolol derivative 002C-3 protects brain against ischemia-reperfusion injury via inhibiting apoptosis and autophagy. Neuroscience Letters. 2015; 588: 178183.

[17] Shu S, Li C, You Y, Qian X, Zhou S, Ling C. Electroacupuncture Ameliorates Cerebral Ischemia-Reperfusion Injury by Regulation of Autophagy and Apoptosis. Evidence-Based Complementary and Alternative Medicine. 2016; 2016: 7297425.

[18] Liu N, Shang J, Tian F, Nishi H, Abe K. In vivo optical imaging for evaluating the efficacy of edaravone after transient cerebral ischemia in mice. Brain Research. 2011; 1397: 66-75.

[19] Liu Y, Zhang T, Xue X, Liu D, Zhang H, Yuan L, et al. Pseudoginsenoside-F11 attenuates cerebral ischemic injury by alleviating autophagic/lysosomal defects. CNS Neuroscience \& Therapeutics. 2017; 23: 567-579.

[20] Jiang Z, Watts LT, Huang S, Shen Q, Rodriguez P, Chen C, et al. The Effects of Methylene Blue on Autophagy and Apoptosis in MRI-Defined Normal Tissue, Ischemic Penumbra and Ischemic Core. PLoS ONE. 2016; 10: e0131929.

[21] Shen P, Hou S, Zhu M, Zhao M, Ouyang Y, Feng J. Cortical spreading depression preconditioning mediates neuroprotection against ischemic stroke by inducing AMP-activated protein kinasedependent autophagy in a rat cerebral ischemic/reperfusion injury model. Journal of Neurochemistry. 2017; 140: 799-813.

[22] Li Q, Zhang T, Wang J, Zhang Z, Zhai Y, Yang G, et al. Rapamycin attenuates mitochondrial dysfunction via activation of mitophagy in experimental ischemic stroke. Biochemical and Biophysical Research Communications. 2014; 444: 182-188.

[23] Bu Q, Liu X, Zhu Y, Liu Y, Wang Y. W007B protects brain against ischemia-reperfusion injury in rats through inhibiting inflammation, apoptosis and autophagy. Brain Research. 2014; 1558: 100108.

[24] Fu L, Huang L, Cao C, Yin Q, Liu J. Inhibition of AMP-activated protein kinase alleviates focal cerebral ischemia injury in mice: 
Interference with mTOR and autophagy. Brain Research. 2016; 1650: 103-111.

[25] Li Y, Zhang J, Chen L, Xing S, Li J, Zhang Y, et al. Ebselen reduces autophagic activation and cell death in the ipsilateral thalamus following focal cerebral infarction. Neuroscience Letters. 2015; 600: 206-212.

[26] Chi OZ, Barsoum S, Vega-Cotto NM, Jacinto E, Liu X, Mellender SJ, et al. Effects of rapamycin on cerebral oxygen supply and consumption during reperfusion after cerebral ischemia. Neuroscience. 2016; 316: 321-327.

[27] Lu T, Jiang Y, Zhou Z, Yue X, Wei N, Chen Z, et al. Intranasal ginsenoside $\mathrm{Rb} 1$ targets the brain and ameliorates cerebral ischemia/reperfusion injury in rats. Biological \& Pharmaceutical Bulletin. 2011; 34: 1319-1324.

[28] Wu M, Zhang H, Kai J, Zhu F, Dong J, Xu Z, et al. Rapamycin prevents cerebral stroke by modulating apoptosis and autophagy in penumbra in rats. Annals of Clinical and Translational Neurology. 2018; 5: 138-146.

[29] Qi Z, Luo Y, Liu X, Wang R, Zhao H, Yan F, et al. AKT/GSK3 $\beta$ dependent autophagy contributes to the neuroprotection of limb remote ischemic postconditioning in the transient cerebral ischemic rat model. CNS Neuroscience \& Therapeutics. 2012; 18: 965-973.

[30] Qi Z, Dong W, Shi W, Wang R, Zhang C, Zhao Y, et al. Bcl2 phosphorylation triggers autophagy switch and reduces mitochondrial damage in limb remote ischemic conditioned rats after ischemic stroke. Translational Stroke Research. 2015; 6: 198-206.

[31] Wang R, Liu Y, Liu X, Jia S, Zhao J, Cui D, et al. Resveratrol protects neurons and the myocardium by reducing oxidative stress and ameliorating mitochondria damage in a cerebral ischemia rat model. Cellular Physiology and Biochemistry. 2014; 34: 854-864.

[32] Jeong JH, Yu KS, Bak DH, Lee JH, Lee NS, Jeong YG, et al. Intermittent fasting is neuroprotective in focal cerebral ischemia by minimizing autophagic flux disturbance and inhibiting apoptosis. Experimental and Therapeutic Medicine. 2016; 12: 3021-3028.

[33] Li L, Chen J, Sun S, Zhao J, Dong X, Wang J. Effects of Estradiol on Autophagy and Nrf-2/are Signals after Cerebral Ischemia. Cellular Physiology and Biochemistry. 2017; 41: 2027-2036.

[34] Lu K, Wang H, Ge X, Liu Q, Chen M, Shen Y, et al. Hyperbaric Oxygen Protects against Cerebral Damage in Permanent Middle Cerebral Artery Occlusion Rats and Inhibits Autophagy Activity. Neurocritical Care. 2019; 30: 98-105.

[35] Gao L, Jiang T, Guo J, Liu Y, Cui G, Gu L, et al. Inhibition of autophagy contributes to ischemic postconditioning-induced neuroprotection against focal cerebral ischemia in rats. PLoS ONE. 2012; 7: e46092.

[36] Qi Z, Yan F, Shi W, Zhang C, Dong W, Zhao Y, et al. AKTrelated autophagy contributes to the neuroprotective efficacy of hydroxysafflor yellow a against ischemic stroke in rats. Translational Stroke Research. 2014; 5: 501-509.

[37] Chen J, Wang X, Chen L, Tang J, Xia Y, Qian K, et al. A sphingosine kinase 2-mimicking TAT-peptide protects neurons against ischemia-reperfusion injury by activating BNIP3-mediated mitophagy. Neuropharmacology. 2020; 181: 108326.

[38] Li C, Li J, Xu G, Sun H. Influence of Chronic Ethanol Consumption on Apoptosis and Autophagy Following Transient Focal Cerebral Ischemia in Male Mice. Scientific Reports. 2020; 10: 6164.

[39] Pan G, Jin L, Shen W, Zhang J, Pan J, Cheng J, et al. Treadmill exercise improves neurological function by inhibiting autophagy and the binding of HMGB1 to Beclin1 in MCAO juvenile rats. Life Sciences. 2020; 243: 117279.

[40] Wang L, Xiong X, Zhang X, Ye Y, Jian Z, Gao W, et al. Sodium Tanshinone IIA Sulfonate Protects against Cerebral Ischemiareperfusion Injury by Inhibiting Autophagy and Inflammation. Neuroscience. 2020; 441: 46-57.

[41] Wang C, Niu F, Ren N, Wang X, Zhong H, Zhu J, et al. Hyperbaric Oxygen Improves Cerebral Ischemia/Reperfusion Injury in Rats Probably via Inhibition of Autophagy Triggered by the Down- regulation of Hypoxia-Inducing Factor-1 Alpha. BioMed Research International. 2021; 2021: 1-8

[42] Vahdati SS, Rezabakhsh A, Taghizadieh M, Khosravi M, Attari MMA, Ghafouri RR. Red Blood Cell Distribution Width Value as a Predictor for Mortality in Stroke Patients. Erciyes Medical Journal. 2020; 42: 317-322.

[43] Sadeghi-Hokmabadi E, Vahdati SS, Rikhtegar R, Ghasempour K, Rezabakhsh A. Public knowledge of people visiting Imam Reza hospital regarding stroke symptoms and risk factors. BMC Emergency Medicine. 2019; 19: 36.

[44] Popa-Wagner A, Dinca I, Yalikun S, Walker L, Kroemer H, Kessler C. Accelerated delimitation of the infarct zone by capillaryderived nestin-positive cells in aged rats. Current Neurovascular Research. 2006; 3: 3-13.

[45] Kalogeris T, Baines CP, Krenz M, Korthuis RJ. Cell biology of ischemia/reperfusion injury. International Review of Cell and Molecular Biology. 2013; 298: 229-317.

[46] Clark SG, Graybeal LL, Bhattacharjee S, Thomas C, Bhattacharya $\mathrm{S}$, Cox DN. Basal autophagy is required for promoting dendritic terminal branching in Drosophila sensory neurons. PLoS ONE. 2018; 13: e0206743.

[47] Xing S, Zhang Y, Li J, Zhang J, Li Y, Dang C, et al. Beclin 1 knockdown inhibits autophagic activation and prevents the secondary neurodegenerative damage in the ipsilateral thalamus following focal cerebral infarction. Autophagy. 2012; 8: 63-76.

[48] Zhang X, Yan H, Yuan Y, Gao J, Shen Z, Cheng Y, et al. Cerebral ischemia-reperfusion-induced autophagy protects against neuronal injury by mitochondrial clearance. Autophagy. 2013; 9: 1321-1333.

[49] Frugier T, Taylor JM, McLean C, Bye N, Beart PM, Devenish RJ, et al. Evidence for the recruitment of autophagic vesicles in human brain after stroke. Neurochemistry International. 2016; 96: 62-68.

[50] Wang X, Fang Y, Huang Q, Xu P, Lenahan C, Lu J, et al. An updated review of autophagy in ischemic stroke: from mechanisms to therapies. Experimental Neurology. 2021; 340: 113684.

[51] Shi R, Weng J, Zhao L, Li X, Gao T, Kong J. Excessive Autophagy Contributes to Neuron Death in Cerebral Ischemia. CNS Neuroscience \& Therapeutics. 2012; 18: 250-260.

[52] Perez-Alvarez MJ, Villa Gonzalez M, Benito-Cuesta I, Wandosell FG. Role of mTORC1 Controlling Proteostasis after Brain Ischemia. Frontiers in Neuroscience. 2018; 12: 60.

[53] Fletcher L, Evans TM, Watts LT, Jimenez DF, Digicaylioglu M. Rapamycin treatment improves neuron viability in an in vitro model of stroke. PLoS ONE. 2013; 8: e68281.

[54] Jiang T, Yu J, Zhu X, Wang H, Tan M, Cao L, et al. Acute metformin preconditioning confers neuroprotection against focal cerebral ischaemia by pre-activation of AMPK-dependent autophagy. British Journal of Pharmacology. 2014; 171: 3146-3157.

[55] Radad K, Moldzio R, Al-Shraim M, Kranner B, Krewenka C, Rausch W. Recent advances in autophagy-based neuroprotection. Expert Review of Neurotherapeutics. 2015; 15: 195-205.

[56] Nabavi SF, Sureda A, Sanches-Silva A, Pandima Devi K, Ahmed T, Shahid M, et al. Novel therapeutic strategies for stroke: the role of autophagy. Critical Reviews in Clinical Laboratory Sciences. 2019; 56: 182-199.

[57] Mehta SL, Kumari S, Mendelev N, Li PA. Selenium preserves mitochondrial function, stimulates mitochondrial biogenesis, and reduces infarct volume after focal cerebral ischemia. BMC Neuroscience. 2012; 13: 79.

[58] Zhang B, Deng F, Zhou C, Fang S. ClC-3 induction protects against cerebral ischemia/reperfusion injury through promoting Beclin1/Vps34-mediated autophagy. Human Cell. 2020; 33: 1046-1055.

[59] Yu S, Yu M, Bu Z, He P, Feng J. FKBP5 Exacerbates Impairments in Cerebral Ischemic Stroke by Inducing Autophagy via the AKT/FOXO3 Pathway. Frontiers in Cellular Neuroscience. 2020; 14: 193.

[60] Zhang Y, He Q, Yang M, Hua S, Ma Q, Guo L, et al. Dichloromethane extraction from Piper nigrum $\mathrm{L}$. and P. longum 
L. to mitigate ischemic stroke by activating the AKT/mTOR signaling pathway to suppress autophagy. Brain Research. 2020; 1749: 147047

[61] Cai Y, Yang E, Yao X, Zhang X, Wang Q, Wang Y, et al. FUNDC1dependent mitophagy induced by tPA protects neurons against cerebral ischemia-reperfusion injury. Redox Biology. 2021; 38: 101792.

[62] Joseph C, Buga A, Vintilescu R, Balseanu AT, Moldovan M, Junker $\mathrm{H}$, et al. Prolonged gaseous hypothermia prevents the upregulation of phagocytosis-specific protein annexin 1 and causes low-amplitude EEG activity in the aged rat brain after cerebral ischemia. Journal of Cerebral Blood Flow and Metabolism. 2012; 32: 1632-1642.

[63] Slevin M, Matou S, Zeinolabediny Y, Corpas R, Weston R, Liu D, et al. Monomeric C-reactive protein-a key molecule driving development of Alzheimer's disease associated with brain ischaemia? Scientific Reports. 2015; 5: 13281.

[64] Liu K, Mo Y, Sun Y. Autophagy and inflammation in ischemic stroke. Neural Regeneration Research. 2020; 15: 1388. 